\title{
NCOA3 Loss Disrupts Molecular Signature of Chondrocytes and Promotes Posttraumatic Osteoarthritis Progression
}

\author{
Bing Zhang ${ }^{\mathrm{a}}$ Zhiyong $\mathrm{Li}^{\mathrm{b}} \quad$ Wei Wang $^{\mathrm{b}}$ Jichao Guo ${ }^{\mathrm{b}}$ Shufeng Kang ${ }^{\mathrm{b}}$ \\ Shizhao Liu ${ }^{\mathrm{b}}$ Hongzhu Li ${ }^{\mathrm{b}}$ Dapeng Wang ${ }^{\mathrm{b}}$ Xiangbei Q ${ }^{\mathrm{b}}$ \\ aDepartment of Hand Surgery, the Third Hospital of Hebei Medical University, Shijiazhuang, \\ 'Department of Orthopaedics, Xiangjiang Branch of the Third Hospital of Hebei Medical University, \\ Shijiazuang, China
}

\section{Key Words}

NCOA3 $・$ Chondrocyte $\cdot$ DNMT1 $・$ Dedifferentiation $•$ Osteoarthritis $\bullet$ NF-KB

\begin{abstract}
Background/Aims: Osteoarthritis (OA) is the most common joint disease. Recently, a novel variant near the nuclear receptor coactivator 3 (NCOA3) has been identified in association with greater risk of developing OA. However, how NCOA3 is regulated in chondrocytes and involved in OA pathogenesis remain elusive. Methods: The expression and DNA methylation of NCOA3 in knee OA cartilage and in vitro dedifferentiated chondrocytes with or without rs6094710 SNP were analyzed by qRT-PCR, immunoblotting, methylation-specific PCR and bisulfite sequencing. NCOA3 was depleted by siRNA or shRNA or inhibited by a chemical inhibitor to assess its role in chondrocyte dedifferentiation or OA pathogenesis in posttraumatic OA animal model established by cruciate ligament transection surgery. Results: We found that compared with normal counterparts, samples with rs6094710 SNP failed to upregulate NCOA3. Further evidence associated this phenotype with DNMT1-mediated hypermethylation in gene promoter region. Moreover, we showed that NCOA3 maintained the molecular signature of chondrocytes dedifferentiating in vitro or exposed to IL-1 $\beta$, nevertheless, NCOA3 appeared dispensable for preventing OA initiation, since NCOA3 loss did not trigger OA in young mice. Instead, NCOA3 loss promoted posttraumatic OA progression, and in parallel, enhanced NF$\mathrm{KB}$ activation. Finally, the promoted posttraumatic OA progression was significantly retarded when administrated with NF-KB pathway inhibitor, suggesting that NCOA3 lose promotes posttraumatic OA at least partially by enhancing NF-KB activation. Conclusion: Thus, our findings indicate a critical role of NCOA3 in chondrocytes, and imply that manipulating NCOA3 might present a potential therapeutic approach to interfere OA progression.
\end{abstract}




\section{Cellular Physiology Cell Physiol Biochem 2018;49:2396-2413 and Biochemistry Published online: 28 September $2018 \begin{aligned} & \text { DO } 2018 \text { The Author(s). Published by S. Karger AG, Basel } \\ & \text { www.karger.com/cpb }\end{aligned}$ \\ Kang et al.: Effects of NCOA3 Loss on Chondrocytes and Osteoarthritis}

\section{Introduction}

Osteoarthritis $(\mathrm{OA})$ is the leading type of arthritis that is estimated to affect over 250 million people worldwide [1]. The therapeutic options for OA are often far from optimal, and its elusive pathogenesis further worsens the status quo [2]. OA is characterized by progressive degeneration of articular cartilage and phonotypical changes of chondrocytes [3]. Biomechanically inferior extracellular matrix would be produced when chondrocytes lose their molecular signature or even die, contributing to cartilage damage and functional loss [4]. In past few years, great efforts have been paid to identify the OA susceptibility genes $[5,6]$. A large meta-analysis of genome wide association studies (GWAS) identified a novel variant rs6094710 near the nuclear receptor coactivator 3 (NCOA3) associated with hip OA occurrence [7]. Specifically, this G/A transition SNP confers to A allele carriers approximate $30 \%$ greater risk of developing hip $\mathrm{OA}$ (odds ratio $1.28 ; \mathrm{P}=7.9 \times 10^{9}$ ) [8].

The protein encoded by $N C O A 3$ is a nuclear receptor coactivator that interacts with nuclear hormone receptors to stimulate their transcriptional activities in a hormonedependent manner [7]. The ensuing studies have shown that the effect of rs6094710 SNP on OA risk depends on reduced expression of NCOA3 [9], which is possibly associated with mechanism of DNA methylation [10]. However, the mechanism of this regulation and how NCOA3 participates in OA pathogenesis are unknown. In this study, we investigated the regulation and function of NCOA3 in chondrocytes and animal OA models. Our findings indicate that its regulation is associated with DNMT1-mediated hypermethylation, and suggest that NCOA3 is involved in maintaining chondrocyte molecular signature in vitro and controlling posttraumatic OA progression in vivo, which may be attributed to its regulation of NF- $\kappa$ B pathway.

\section{Materials and Methods}

\section{Patients}

Human articular chondrocytes of knee joint were isolated from OA patients who underwent knee replacement at Third Hospital of Hebei Medical University (OA group). The OA patients met the American College of Rheumatology classification criteria for OA diagnosis [11]. The OA degree was evaluated using Kellgren and Lawrence (KL) grading scale [12]. Normal articular chondrocytes of knee joint, as evaluated and confirmed with histological analysis, were isolated from patients with amputation from accidents (nonOA group). The patients in OA group and non-OA group were similar in age and sex. Informed consent was obtained from each patient. The cell isolation procedure and the informed consent were all in accordance with the terms of the Medical Ethical Committee of Third Hospital of Hebei Medical University. More specific clinical information regarding the recruited 30 OA patients and 42 non-OA patients is provided in Table S1 (for all supplementary material see www.karger.com/10.1159/000493839/).

\section{SNP genotyping analysis}

When sampling human chondrocytes, a part of these cells for each patient were immediately stored at $-80^{\circ} \mathrm{C}$ for further extraction of genomic DNA. The genomic DNA of each sample was analyzed in triplicate. Pyrosequencing technique was used to genotype SNP rs6094710. The primers used for SNP rs6094710 were designed to reside within single exon. The primer sequences (5'-3') were listed as follows: forward: biotin-GATTTTTCACTGGGGATGGG; reverse: CAAGTGAGCACGTACACAACTCC; sequencing: CACAACTCCAATAAACACAT. The genotype SNP rs6094710 of each patient is provided in Table S2.

\section{Chondrocyte isolation and in vitro dedifferentiation culture}

Human articular chondrocytes were isolated from non-OA patients as described before [13]. Chondrocytes with or without SNP rs6094710 were randomly selected from donors according to the recorded genotype. For isolating chondrocytes, cartilage tissues were dissected from the knee joint explant surfaces and rinsed in sterile saline buffer solution. In a sterile biosafety cabinet, the cartilage tissues were cut into small pieces through using a sterile surgical blade. Cartilage explants were incubated with $2 \mathrm{mg} /$ 


\section{Cellular Physiology Cell Physiol Biochem 2018;49:2396-2413

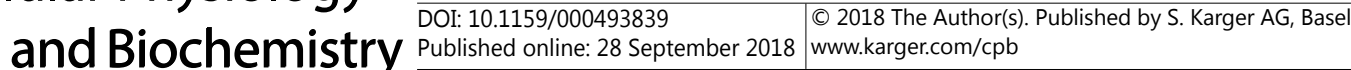 \\ Kang et al.: Effects of NCOA3 Loss on Chondrocytes and Osteoarthritis}

$\mathrm{ml}$ pronase solution (Sigma) for $120 \mathrm{~min}$ at $37^{\circ} \mathrm{C}$ and then fully digested overnight at $37^{\circ} \mathrm{C}$ in $1 \mathrm{mg} / \mathrm{ml}$ collagenase II solution (Sigma) under continuous agitation. The obtained cell suspensions were filtered through a $70 \mu \mathrm{m}$ cell strainer (BD Biosciences) and the resulting cells were seeded in culture plate with a density of 25,000 cells $/ \mathrm{cm}^{2}$ and further cultured in a humidified incubator at $37^{\circ} \mathrm{C}$ with $5 \% \mathrm{CO}_{2}$. The culture medium was DMEM/F12 (Invitrogen) supplemented with 10\% fetal bovine serum (FBS) (Invitrogen), 1\% penicillin-streptomycin (Invitrogen) and 1\% L-glutamine (Invitrogen). Chondrocytes were cultured in monolayer throughout. When cell density was reaching full confluence, cells were passaged by 1:3 ratio and cultured for up to passage 4, 6 or 8 at the request of experimental purposes.

\section{Animals}

Animal experiments were performed with male C57BL/6J wild-type mice. All experiments using animals were in accordance with protocols approved by the Institutional Animal Care and Use Committees of Third Hospital of Hebei Medical University for animal welfare. 8-week-old male C57BL/6 wild-type mice were maintained under specific pathogen-free conditions, and randomly divided into different groups by age and weight prior to experiments.

\section{Antibodies and reagents}

The antibodies and reagents were purchased from the following sources: NCOA3 (F-2) (Santa Cruz, sc5305), GAPDH (0411) (Santa Cruz, sc47724), COL2A1 (M2139) (Santa Cruz, sc52658), Aggrecan (6-B4) (abcam, ab3778), COL1A1 (3G3) (Santa Cruz, sc293182), p65 (abcam, ab16502), phospho-p65 (S536) (abcam, ab28856), IкB $\alpha$ (C-21) (Santa Cruz, sc371), phospho-IкB $\alpha$ (Ser32/36) (Cell Signaling, \#9246), Goat anti-rabbit IgG-HRP (Santa Cruz, sc-2004), Goat anti-mouse IgG-HRP (Santa Cruz, sc-2302). 5-Azacytidine (Sigma, A2385), Bufalin (Sigma, B0261), BrdU (5-Bromo-2'-deoxyuridine) (Millipore, \#19-160), ML120B (Sigma, SML1174), recombinant IL-1 $\beta$ (R\&D System, NP-000567).

\section{In vitro treatment and transfection for chondrocytes}

For 5-Aza treatment, freshly isolated human articular chondrocytes were cultured as described before and treated with DMSO or $1 \mu \mathrm{M}$ 5-Aza for periods of two population doublings. For bufalin treatment, chondrocytes were treated with DMSO or increasing concentrations of bufalin $(1,2,5,10 \mathrm{nM})$ for 72 hours. For IL-1 $\beta$ treatment, chondrocytes were treated with vehicle or IL-1 $\beta(0,1,2 \mathrm{ng} / \mathrm{ml})$ for 24 hours. Small interfering RNA transfection assay was performed with $40 \mathrm{nM}$ siRNA and reagent lipofectamine RNAimax (Invitrogen) for 72 hours according to the manufacturer's instructions. siRNA targeting luciferase with same concentration was used as negative control.

\section{Methylation-specific PCR and bisulfite sequencing}

Genomic DNA was extracted from human chondrocytes and modified with bisulfite. Briefly, $2 \mu \mathrm{g}$ of genomic DNA was denatured in $0.2 \mathrm{M} \mathrm{NaOH}$ at $37^{\circ} \mathrm{C}$ for $10 \mathrm{~min}$ and then treated with $10 \mathrm{mM}$ hydroquinone and $3 \mathrm{M}$ sodium bisulfite ( $\mathrm{pH}$ 5.0) (Sigma, \#243973) at $50^{\circ} \mathrm{C}$ for further 16 hours. Modified DNA was analyzed with methylation-specific PCR (MSP) primers for NCOA3. The PCR products were electrophoresed on a $2 \%$ agarose gel, stained with ethidium bromide and visualized under ultraviolet illuminator.

To examine the methylation status of $38 \mathrm{CpG}$ dinucleotides within the promoter region of $\mathrm{NCOA}_{3}$ gene, the NCOA3 gene promoter was amplified from the bisulfite-modified DNA by two rounds of PCR using nested primers specific to the bisulfite-modified sequence of the NCOA3 gene CpG islands. The PCR products were cloned into the PCR II vector through the TOPO-TA cloning kit (Invitrogen) in accordance with manufacturer's instruction. Plasmid DNA was isolated from 5 randomly selected clones using the QIAprep Spin Miniprep Kit (Qiagen) and prepared for automated DNA sequencing analysis. The reaction conditions were set as follows: $95^{\circ} \mathrm{C}$ for 10 seconds, $50^{\circ} \mathrm{C}$ for 5 seconds, $60^{\circ} \mathrm{C}$ for 4 min for 25 cycles. DNA was sequenced using dye terminator technology and an ABI 3730xl sequencer (Applied Biosystems). The positions and sequences of the primers mentioned above are shown in Table S2.

Adenoviral vector construction for short hairpin RNA targeting NCOA3

The target sequence of shNCOA3 is (5'-CCGGGCAGACTCCTTAGGACCGCTTCTCGAG AAGCGGTCCTAAGGAGTCTGCTTTTTG-3') according to previous study [14]. A non-specific scrambled shRNA sequence (5'-CCGGCAACAAGATGAAGAGCACCAACTCGAGTTGGTGCTCTTCATCTTGTTGTTTTT-3') was used 


\section{Cellular Physiology Cell Physiol Biochem 2018;49:2396-2413

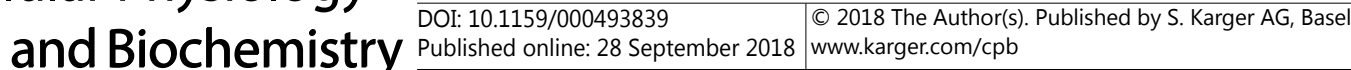 \\ Kang et al.: Effects of NCOA3 Loss on Chondrocytes and Osteoarthritis}

as negative control throughout this study. The above sequences were invidiously ligated into the shuttle vector 1.0-CMV (Ambion) and confirmed by DNA sequence. The plasmid was co-transfected into human embryonic kidney (HEK) 293 cells with Lipofectamine 2000 reagent (ThermoFisher Scientific). After 48 hours, adenovirus particles in culture medium were collected and purified with Adenovirus Purification Kits (Takara), the resulting adenovirus solutions were stored at $-80{ }^{\circ} \mathrm{C}$ until used in experiment.

\section{Histological analysis}

Both right and left knees from treated 8-week-old male wild-type C57Bl/6 mice were fixed overnight at $4{ }^{\circ} \mathrm{C}$ in $4 \%$ formaldehyde solution, then decalcified for 3 weeks in $0.5 \mathrm{M}$ EDTA (pH 7.5), embedded in paraffin, and $5 \mathrm{~mm}$ thick sections were prepared. Hematoxylin-Safranin 0 staining was performed as previously described [15]. The severity degree of cartilage damage was assessed through histological score of knee sections stained with Hematoxylin-Safranin 0 by an experienced investigator according to OARSI guidelines [16], who was blinded to the grouping of mice. The severity degree of lesion (0-6) was scored on frontal knee sections. 0 : intact surface and cartilage morphology; 1 : small fibrillations without cartilage loss; 2: vertical clefts below superficial layer and some loss of surface lamina; 3-6: extension of vertical clefts/ erosions to cartilage ( $3:<25 \% ; 4: 25-50 \% ; 5: 50-75 \%$ and $6:>75 \%)$. The final score indicated the mean of the tibial scores from the medial and lateral side.

\section{Cruciate ligament transection surgery}

CLT and sham surgeries for 8-week-old male C57BL/6J wild-type mice were performed as previously described [17]. Briefly, mice were anesthetized with intraperitoneal injection of $200 \mu$ l volume of $1.2 \%$ tribromoethanol (Sigma). The skin around knees was denuded and cleaned with surgical soap and washed with $70 \%$ alcohol for three times before surgery. About $5-\mathrm{mm}$ incision in the medial side of the joint parallel to the patellar tendon was made by a Feather \#11 scalpel (Feather Safety Razor). Then, a 0.90-mm 20-gauge microsurgical scalpel (Beaver-Visitec) was inserted into the knee joint space and transected the anterior and posterior cruciate ligaments (CLT surgery). No transection was performed in another group of mice (sham surgery). The cut skin was closed by 5.0 monofilament sutures (Ethicon). Researchers were blind to the grouping of mice when surgery was performed.

\section{Intra-articular injection}

Mice were transiently anesthetized with $3 \%$ isoflurane. Knee joint area was shaved clean. adenoviral vector expressing shCtrol or shNCOA3 was diluted in $5 \mu$ l sterile PBS and injected through a $25 \mu$ l CASTIGHT syringes (Hamilton Company) and 33-gauge needles (Hamilton Company). For inhibitor injection experiments, we selected the minimum effective concentration of bufalin that showed to considerably inhibit NCOA3 according to our pilot studies. Bufalin $(1 \mathrm{mg} / \mathrm{kg})$ or equal volume of DMSO was injected intraarticularly into the knee of mice three times per week until the end of the experiments.

\section{Western blotting}

Chondrocytes were harvested and washed three times by PBS, and then lysed using RIPA buffer with protease inhibitors (Sigma) on ice for $10 \mathrm{~min}$. Protein samples were boiled in 1×loading buffer and resolved by SDS-PAGE and transferred onto PVDF membrane (Millipore), which was blocked by 5\% skimmed milk (BD Difco) in TBS supplemented with $0.1 \%$ Tween (TBST) before probing with primary antibodies at $4^{\circ} \mathrm{C}$ overnight. Membranes were washed with TBST for three times and then incubated with secondary antibodies at room temperature for 1 hour. After being washed in TBST for three times, membranes were subjected to the enhanced chemiluminescence (GE Healthcare, RPN2209) for protein detection by GE ImageQuant LAS 4000 instrument. Western bands were quantified using ImageJ software.

\section{RNA purification and Real-time Quantitative polymerase chain reaction}

Tissue or cell homogenization and the subsequent total RNA isolation with Trizol-based method (Thermo Fisher Scientific, \#15596026) were performed according to the manufacturer's instructions. mRNA level of interests was quantified by SYBR green real-time PCR kit (TakaRa, \#RR420A) with the 7500 Real-Time PCR System (Applied Biosystems, USA). Data were analyzed using the comparative Ct method with $G A P D H$ as endogenous control throughout. Sequences of primers probing human or mouse genes are listed in Table S3. 


\section{Cellular Physiology Cell Physiol Biochem 2018;49:2396-2413 and Biochemistry \begin{tabular}{l|l} 
DOI: 10.1159/000493839 & $\begin{array}{l}\text { (c) } 2018 \text { The Author(s). Published by S. Karger AG, Basel } \\
\text { www.karger.com/cpb }\end{array}$
\end{tabular} \\ Kang et al.: Effects of NCOA3 Loss on Chondrocytes and Osteoarthritis}

Chromatin immunoprecipitation assay

Chondrocytes were harvested and washed three times by PBS, and then cross-linked with $1 \%$ formaldehyde for $10 \mathrm{~min}$ at room temperature and processed according to the procedures described in ChIP Assay Kit (Millipore, \#17-295). anti-DNMT1 antibody (abcam, \#ab13537,) or normal mouse IgG1 (abcam, \# ab91353) was used. Antibody-chromatin complexes were pulled-down using magnetic protein G beads (Invitrogen, \#10003D), washed, eluted, reverse-crosslinked, digested with proteinase K and analyzed by Real-time quantitative PCR. ChIP primers were listed as follows:

NCOA3-\#1:F:5'-cgatcacttggtaagggggtgcctg-3';R:5'-cgaactgaggcacaaggtcggaagc-3';

NCOA3-\#2: F: 5'-ctcatctgaaaaatgaggatga-3'; R: 5'-cgctttcgcccagcatcgactggg-3';

NCOA3-\#3: F:5'-gccgcacgagccacgtcgccacg-3';R:5' ccgctgccgtcgctgtggagcggc-3'.

\section{BrdU incorporation, apoptosis detection and flow-cytometry analysis}

Chondrocytes were harvested and washed twice and resuspended in PBS. Chondrocytes were analyzed by BrdU incorporation using the FITC BrdU Flow Kit (BD Pharmingen) for measuring proliferation, or double stained with FITC-conjugated annexin-V monoclonal antibody and PI dye for detecting apoptosis using the Annexin V-FITC Apoptosis Detection kit (BD Biosciences) according to the manufacturer's instructions. After staining and washing, cells were measured by flow cytometry analyzer (BD, LSRFortessa.).

\section{Statistical analysis}

All statistical analyses were performed with GraphPad Prism 6 software. Data are representative of at least 3 independent experiments and expressed as mean \pm s.d. Statistical analysis comparing data of two groups was assessed by Student's t test, and statistical analysis comparing data between multiple groups was performed by one-way ANOVA followed by Tukey's post-hoc. A value of $\mathrm{P}<0.001, \mathrm{P}<0.01$ or $\mathrm{P}<0.05$ indicates a statistically significant difference and NS represents no significant difference.

\section{Results}

Knee OA patients carrying rs6094710 SNP fail to upregulate NCOA3 in cartilage

The single nucleotide polymorphism (SNP) rs6094710, one locus at 20q13 near the NCOA3 gene, was initially identified as a novel variant associated with hip $0 \mathrm{~A}$, and meanwhile NCOA3 expression was found to be significantly reduced in OA cartilage [7]. However, paradoxically, one study showed that NCOA3 was upregulated in hip OA cartilage compared with non-OA cartilage [9]. Regardless of this contradiction, another study demonstrated that the expression of rs6094710 was reduced in both hip OA as well as knee OA tissues [18]. Here, to provide a further line of evidence on NCOA3 expression in response to OA affliction, we compared its expression between non-OA and $\mathrm{OA}$ knee cartilages stratified by genotype at rs6094710 SNP. The results turned out that compared with their non-OA counterparts, NCOA3 expression was significantly upregulated both at mRNA level $(1.67$ fold, $p=0.0012)$ (Fig. 1A) and protein level (2.12 fold, $\mathrm{p}=0.0169)$ (Fig. 1B-1C) in OA patients without rs6094710 SNP, whereas, NCOA3 remained nearly steady in OA patients with rs6094710 SNP both at mRNA level $(p=0.4211)$ (Fig. 1D) and protein level $(p=0.3820)$ (Fig. 1E-1F), even though all of the recruited patients in our study were actually heterozygotes for the allele of SNP, carrying a single G/A transition, possibly due to its rare minor allele frequency (MAF) [7]. Thus, inconsistent with previous report [18], we did not found reduced NCOA3 expression in knee OA cartilage with rs6094710 SNP. This discrepancy may be attributed to different sources of utilized control cartilage and/or sampling size. At any rate, our results imply that NCOA3 may not be normally upregulated in knee OA cartilage with rs6094710 SNP, which would otherwise occur in knee OA cartilage without this SNP. 


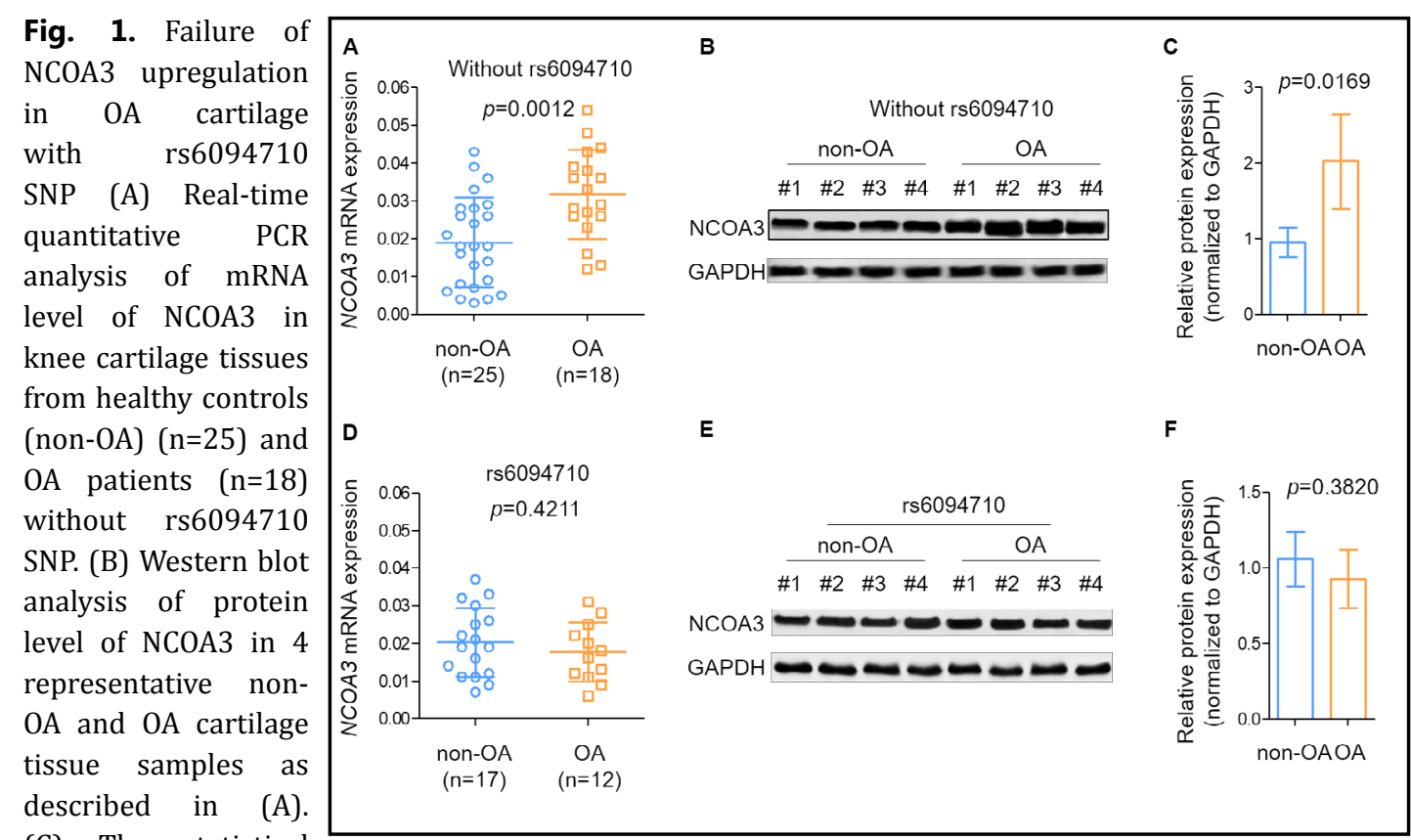

(C) The statistical

analysis of band intensity of results shown in (B). (D) Real-time quantitative PCR analysis of mRNA level of NCOA3 in knee cartilage tissues from healthy controls (non-OA) $(n=17)$ and OA patients $(n=12)$ with rs6094710 SNP. (E) Western blot analysis of protein level of NCOA3 in 4 representative non-OA and OA cartilage tissue samples as described in (D). (F) The statistical analysis of band intensity of results shown in (E). In (A) and (D), The results were normalized to human GAPDH. Each symbol represented the mean value of 3 replicates for each sample. In (B) and (E), Human GAPDH was used as loading control. Experiments were conducted at three times independently and shown here were the representative images. In (C) and (F), The protein expression in each sample was normalized to human GAPDH and the results relative to nonOA group were shown. The statistical analysis was performed using Student's $t$ test. Data are mean \pm s.d.

Dedifferentiated chondrocytes with rs6094710 SNP cannot upregulate NCOA3 and show enhanced OA-like molecular signature in vitro

We next sought to examine whether the expression pattern of NCOA3 in cartilage could also be found in chondrocytes with dedifferentiated status, one hallmark of OA pathology [19]. We isolated healthy human knee articular chondrocytes with or without rs6094710 SNP, and then cultivated and continuously passaged these cells in monolayer status, for the purpose of mimicking the dedifferentiation process occurring in OA cartilage, during which the normal cellular characteristics would gradually vanish [20]. Surprisingly, the results showed that during the dedifferentiation culture from passage 2 to passage 8 , as validated by expression lose of chondrocyte markers such as type 2 collagen (COL2A1) and aggrecan and concomitantly increased expression of fibroblast marker type 1 collagen (COL1A1) [21], the expression of NCOA3 was gradually induced up to approximately twofold both at mRNA level (Fig. 2A) and protein level (Fig. 2B-2C) in chondrocytes without rs6094710 SNP. In stark contrast, NCOA3 expression could not be upregulated in chondrocytes with rs6094710 SNP, even though these cells were successfully dedifferentiated into OA-like phenotype during culture (Fig. 2D-2F). These results are consistent with the above evidence we obtained from corresponding clinical samples (Fig. 1). Moreover, another striking observation was the significantly enhanced OA-like molecular characteristics exhibited by chondrocytes with rs6094710 SNP compared with normal ones (Table S4), as shown by more accelerated loss of COL2A1 and aggrecan and gain of COL1A1. These results suggest a critical role of normally upregulated NCOA3 expression in maintaining the molecular signature of chondrocytes. 


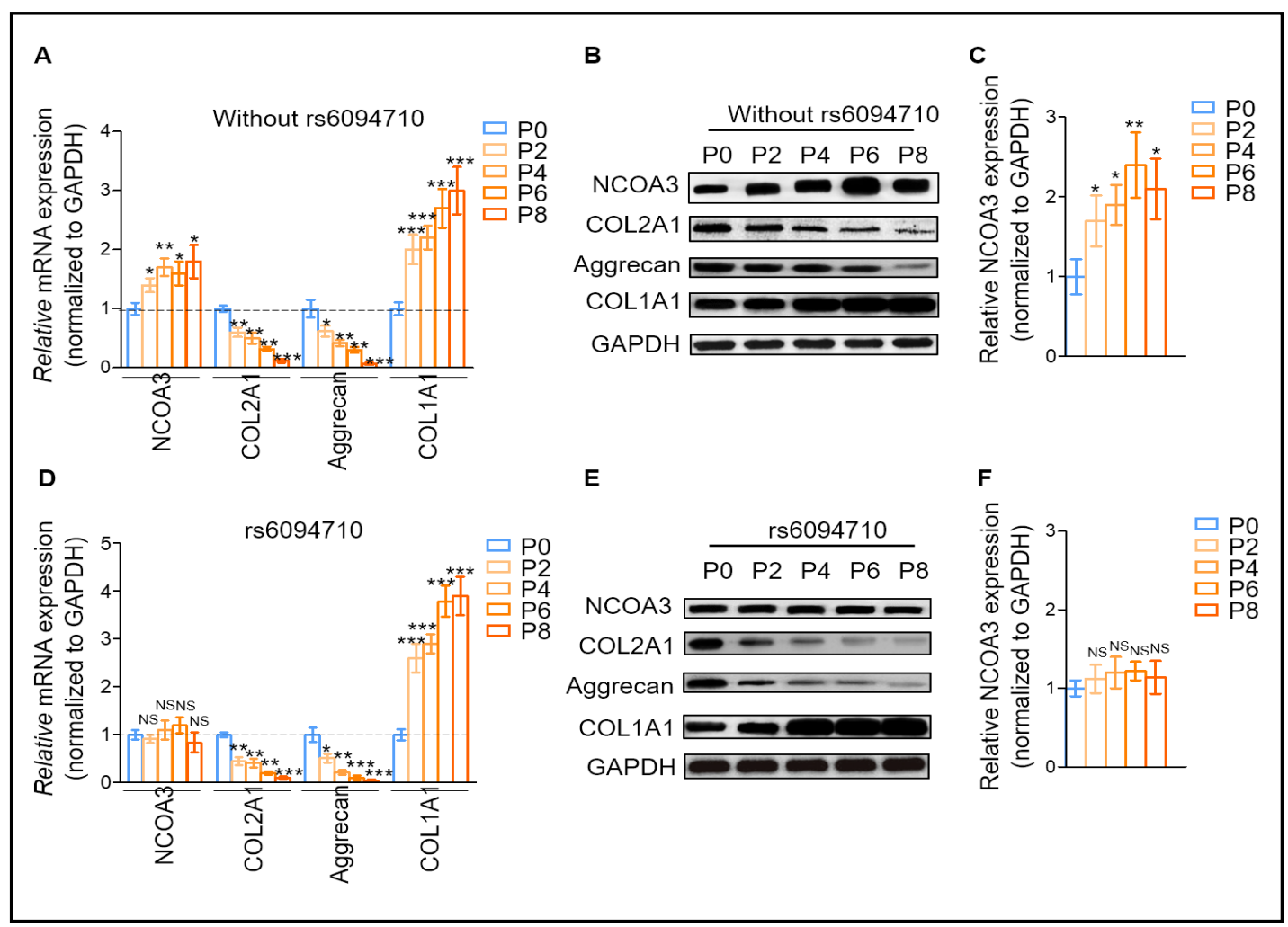

Fig. 2. Chondrocytes with rs6094710 SNP cannot upregulate NCOA3 and display enhanced OA-like molecular signature in dedifferentiation culture (A) Real-time quantitative PCR analysis of mRNA level of NCOA3, COL2A1, Aggrecan and COL1A1 in dedifferentiating chondrocytes without rs6094710 SNP from passage 0 (P0) to passage 8 (P8) as indicated. (B) The chondrocytes in each passage as described in (A) were collected and analyzed by Western blot for detecting proteins of NCOA3, COL2A1, Aggrecan and COL1A1. (C) The statistical analysis of band intensity of Western blot results shown in (B). (D) Real-time quantitative PCR analysis of mRNA level of NCOA3, COL2A1, Aggrecan and COL1A1 in dedifferentiating chondrocytes with rs6094710 SNP genotype from passage 0 (P0) to passage 8 (P8) as indicated. (E) The chondrocytes in each passage as described in (D) were collected and analyzed by Western blot for detecting proteins of NCOA3, COL2A1, Aggrecan and COL1A1. (F) The statistical analysis of band intensity of Western blot results shown in (E). In (A) and (D), Expression level of each gene was normalized to human GAPDH and the results of each passage were compared to those of P0. Each column represents the mean value of 3 replicates for each sample and the results were representative of 4 chondrocyte donors. In (B) and (E), Human GAPDH was used as loading control. Experiments were conducted at three times independently and shown here were the representative images. In (C) and (F), The protein expression in each sample was normalized to human GAPDH and the results relative to P0 group were shown. The statistical analysis was performed using a one-way ANOVA with Tukey post hoc test. Data are mean \pm s.d. ${ }^{* * *}, \mathrm{P}<0.001 ; * *, \mathrm{P}<0.01 ;{ }^{*}, \mathrm{P}<0.05$; NS, not significant.

\section{NCOA3 induction failure caused by rs6094710 SNP is associated with DNMT1-mediated hypermethylation}

One previous study has suggested that NCOA3 gene transcription could be controlled by DNA methylation process which appears irregular when rs6094710 SNP exists [10]. This clue prompted us to investigate whether the NCOA3 deregulation in chondrocytes with rs6094710 SNP is associated with abnormal DNA methylation. First, we compared the methylation alteration of NCOA3 gene promoter in chondrocytes with or without rs6094710 SNP when dedifferentiating in vitro. As shown in Fig. 3A, the NCOA3 gene promoter was hypermethylated in freshly isolated healthy chondrocytes ( $\mathrm{P} 0$ passage), and after 6 passages of dedifferentiation, it was largely converted to unmethylated status in chondrocytes without 


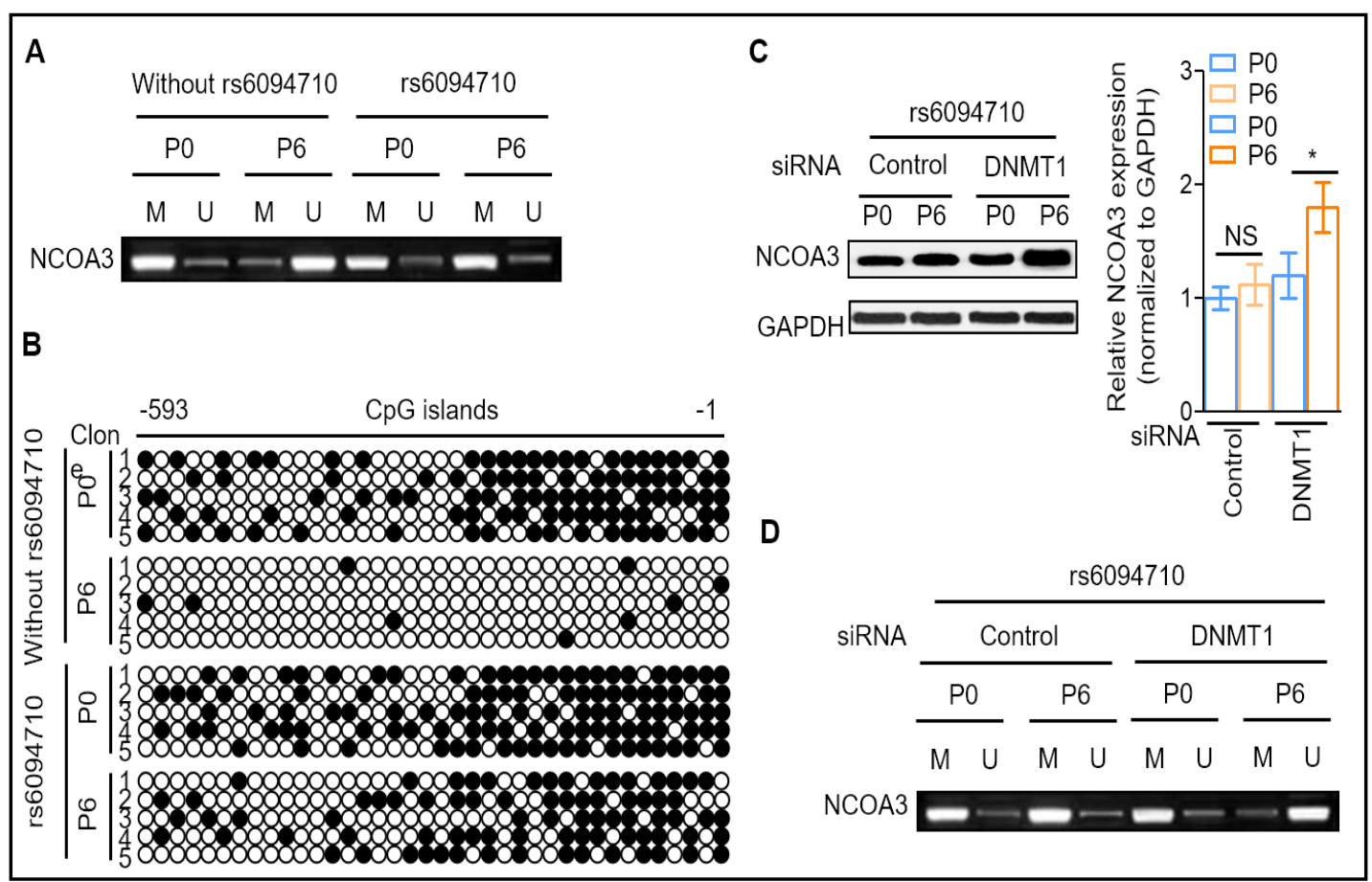

Fig. 3. NCOA3 upregulation failure caused by rs6094710 SNP is partially attributed to DNMT1-mediated hypermethylation (A) Genomic DNA extracted from P0 and P6 chondrocytes with or without rs6094710 was treated with bisulfite and analyzed by methylation-specific PCR (MSP) using the methylated DNA (M)and unmethylated DNA (U)-specific primers for human NCOA3. (B) PCR products from bisulfite-treated genomic DNA were sequenced for the predicted $38 \mathrm{CpG}$ sites located between nucleotides -593 and -1 of the NCOA3 promoter. The horizontal dots represent $\mathrm{CpG}$ islands and the vertical dots represent the sequenced individual five clones. Black dot represents a methylated and white dot represents an unmethylated cytosine residue within the CpG islands. (C-D) P0 and P6 chondrocytes with rs6094710 SNP were transfected with siCtrl or siDNMT1 for 3 days. The protein level of NCOA3 was measured by Western blot analysis (C). GAPDH was used as loading control. The experiments were conducted for 3 times independently. The representative image was shown (Left). The band intensity of Western blot results was statistically analyzed. The protein expression in each sample was normalized to human GAPDH and the result relative to P0 group was shown (Right). Genomic DNA was treated with bisulfite and analyzed by MSP using the methylated DNA (M)- and unmethylated DNA (U)-specific primers for human NCOA3 (D). The statistical analysis was performed using Student's t test. Data are mean \pm s.d. ${ }^{*}, \mathrm{P}<0.05$; NS, not significant.

rs6094710 SNP, indicating its activated transcription, which is in line with the results of Fig. 2A-2C. However, the methylation extent of NCOA3 gene promoter in chondrocytes with rs6094710 SNP remained nearly unchanged, this reflects its stagnant transcriptional activity and is consistent with its failed induction shown in Fig. 2D-2F. In addition, we also examined the total of $38 \mathrm{C}_{\mathrm{p}} \mathrm{G}$ sites spanning from nucleotides -593 to -1 within NCOA3 promoter by bisulfite sequencing. Highly condensed cytosine methylation could be observed in the $C_{p} G$ islands of NCOA3 promoter in P0 passage chondrocytes, which was almost reversed in P6 passage chondrocytes without rs6094710 SNP (Fig. 3B upper half). Likewise, a large part of the $\mathrm{C}_{\mathrm{p}} \mathrm{G}$ islands of NCOA3 promoter in P6 passage chondrocytes with rs6094710 SNP maintained in highly methylated status, even though the whole methylated extent appeared relieved moderately compared with P0 passage cells (Fig. 3B lower half). These results suggest that the aberrant DNA methylation process seen in the $\mathrm{C}_{\mathrm{p}} \mathrm{G}$ islands of NCOA3 promoter in chondrocytes with rs6094710 SNP is possibly associated with the failure of induction of NCOA3 expression when OA or OA-like pathology emerges. 


\section{Cellular Physiology Cell Physiol Biochem 2018;49:2396-2413

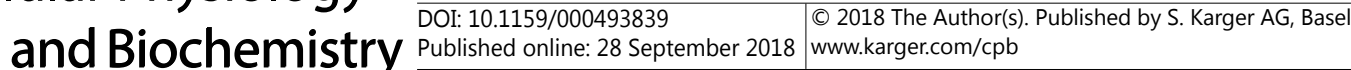 \\ Kang et al.: Effects of NCOA3 Loss on Chondrocytes and Osteoarthritis}

DNA methylation decoration is mediated by DNA methyltransferases (DNMTs), and in mammalian cells, three catalytically active DNMTs perform this role, including DNMT1, DNMT3A and DNMT3B [22]. Therefore, to identify the regulator(s) which may be responsible for rendering NCOA3 promoter in hypermethylation status in chondrocytes with rs6094710 SNP, we then examined the effect of small interfering RNA (siRNA) targeting individual DNMTs on NCOA3 gene expression and methylation. Compared with siCtrl treatment, the mRNA level of NCOA3 was significantly induced in P6 passage chondrocytes with rs6094710 SNP when DNMT1 was silenced, however, in DNMT3A or DNMT3B-silenced group, NCOA3 expression was almost unaffected (Fig. S1A). Consistent with these transcriptional results, the NCOA3 protein level was also upregulated upon DNMT1 siRNA transfection (Fig. 3C), which is similar to the observations found in chondrocytes without rs6094710 SNP (Fig. 2A-2C). Furthermore, DNMT1 deficiency largely reverted the NCOA3 gene promoter to unmethylated status in chondrocytes with rs6094710 SNP after 6 passages dedifferentiation (Fig. S1B), and accordingly, compared with the results of Fig. 3B, the $\mathrm{C}_{\mathrm{p}} \mathrm{G}$ islands of NCOA3 promoter in these cells became obviously unmethylated, although the degree of unmethylation seemed less than that of cells without rs6094710 SNP (Fig. S1B). These evidence clearly indicate that the aberrant expression pattern of NCOA3 in chondrocytes carrying rs6094710 SNP is at least attributed to DNMT1-mediated hypermethylation on gene promoter region. In fact, another supportive evidence showed that DNMT1 was more tightly bound to NCOA3 promoter regions in cells with rs6094710 SNP compared with normal cells (Fig. S1C). Intriguingly, as shown in Fig. S1C, the interaction between DNMT1 and NCOA3 promoter was concentrated on proximal region, and the binding intensity maintained nearly unchanged in cells with rs6094710 SNP during dedifferentiation, which would otherwise gradually faded in normal cells.

To further strengthen our conclusion, we used 5-Azacytidine (5-AZa), a common inhibitor agent of DNMT1, for demethylating DNA [23]. As expected, 5-Aza treatment at least partially recovered the inducible effect of in vitro dedifferentiation on NCOA3 expression at both mRNA level (Fig. S1D) and protein level (Fig. S1E). Overall, the above results suggest that the induction failure of NCOA3 caused by rs6094710 SNP is associated with dysregulated methylation process, where DNMT1 plays a critical role.

NCOA3 upregulation maintains molecular signature of chondrocytes during dedifferentiation or exposed to $I L-1 \beta$

As suggested by the results of Fig. 2, normally upregulated NCOA3 may have a protective role in sustaining molecular signature of chondrocytes. Besides, considering NCOA3 can exert transcriptional activities [24-27], we speculated that NCOA3 may be involved in controlling the transcriptional program of chondrocyte differentiation. To validate this function of NCOA3, we knockdown its expression with specific siRNA in normal chondrocytes during in vitro dedifferentiation. Indeed, compared with siCtrl, NCOA3-knockdown cells showed enhanced OA-like molecular characteristics, as evidenced by more accelerated loss of COL2A1 and aggrecan and gain of COL1A1 both at mRNA level (Fig. 4A) and protein level (Fig. 4B), phenocopying the results of Fig. 2D-2F. Additionally, the OA-like dedifferentiation was also enhanced when cells were treated with bufalin, a potent chemical inhibitor of NCOA3, which efficiently reduces NCOA3 protein level posttranscriptionally [28, 29] (Fig. S2A-S2B). Moreover, NCOA3 knockdown promoted catabolic events in chondrocytes exposed to IL$1 \beta$ (Fig. 4C-4D), which is a proinflammatory cytokine that promotes catabolism in arthritic cartilage [30]. Collectively, these results not only support an important role of NCOA3 in preventing OA-like dedifferentiation and catabolic events driven by IL-1 $\beta$ in vitro, but also provide a hint for explaining the association between rs6094710 risk allele and OA.

\section{NCOA3 inhibition alone cannot trigger OA in young mice}

To investigate whether inhibiting NCOA3 triggers OA in vivo, we suppressed its expression in local cartilage by intra-articular delivery of the adenoviral vector-mediated NCOA3specific shRNA into knees of 8-week-old mice. As shown, NCOA3 expression was effectively 
Fig. 4. NCOA3 induction maintains chondrocyte molecular signature in dedifferentiation culture or exposed to IL-1 $(\mathrm{A}) \quad$ Real-time quantitative PCR analysis of mRNA level of NCOA3, COL2A1, Aggrecan and COL1A1 in dedifferentiating c h o n d r o c y t e s transfected with siCtrl or siNCOA3 from passage 0 (P0) to passage 4 (P4). Expression level of each gene was normalized to human GAPDH. The results of each passage were compared to those of $\mathrm{PO}$ or the result of siNCOA3

was compared to that of siCtrl in each passage as indicated. Each column represents the mean value of 3 replicates for each sample and the results were representative of 3 chondrocyte donors. (B) Western blot analysis of protein level of NCOA3, COL2A1, Aggrecan and COL1A1 in chondrocytes as prepared in (A). Human GAPDH was used as loading control. Experiments were conducted at three times independently. The representative image was shown. (C-D) Chondrocytes transfected with siCtrl or siNCOA3 were treated with increasing concentration of IL-1 $\beta$ as indicated for 24 hours. The mRNA level (C) and protein level (D) of NCOA3, COL2A1, Aggrecan and COL1A1 were measured by real-time quantitative PCR analysis and Western blot analysis. GAPDH was used as reference and loading control. Each column represents the mean value of 3 replicates for each sample and the results were representative of 3 experiments. The statistical analysis was performed using a one-way ANOVA with Tukey post hoc test. Data are mean \pm s.d. ***, P<0.001; **, $\mathrm{P}<0.01 ;{ }^{*}, \mathrm{P}<0.05$.

abrogated at 2 and 4 weeks after injection (Fig. S3A). However, no obvious cartilage damage was caused by NCOA3 loss at these two time points compared with shCtrl group, as shown by Hematoxylin-Safranin 0 staining (Fig. S3B) and evaluated by histological OARSI grading scale ( $p=0.2857$ at 4 weeks; $p=0.3603$ at 6 weeks) (Fig. S3C). In addition to inhibiting NCOA3 expression by shRNA targeting, intra-articular injection of bufalin into mouse knees could not cause cartilage damage or evoke OA either (Fig. S3D-S3F). Taken together, these data suggest that the loss of NCOA3 alone cannot initiate OA at least in young mice.

\section{NCOA3 inhibition promotes posttraumatic OA in vivo}

Considering that NCOA3 may possess some protective roles against OA or OA-like pathology, and the evidence indicating that NCOA3 loss alone is not sufficient for OA initiation, we therefore speculated NCOA3 may exert its effect during OA progression. To test this possibility, we applied a posttraumatic knee OA animal model which was established by the cruciate ligament transection (CLT) surgery [31], which imitates the posttraumatic $\mathrm{OA}$ in humans commonly caused by anterior cruciate ligament tearing. Likewise, we still used the adenoviral vector-mediated shRNA to efficiently inhibit NCOA3 expression in local cartilage (Fig. 5A). Compared with sham surgery control (sham), CLT group showed obvious damage in cartilage (Fig. 5B) and higher OARSI grade (Fig. 5C) at both 4 and 6 weeks after surgery. Remarkably, when NCOA3 was absent, the cartilage damage was more severe (Fig. 


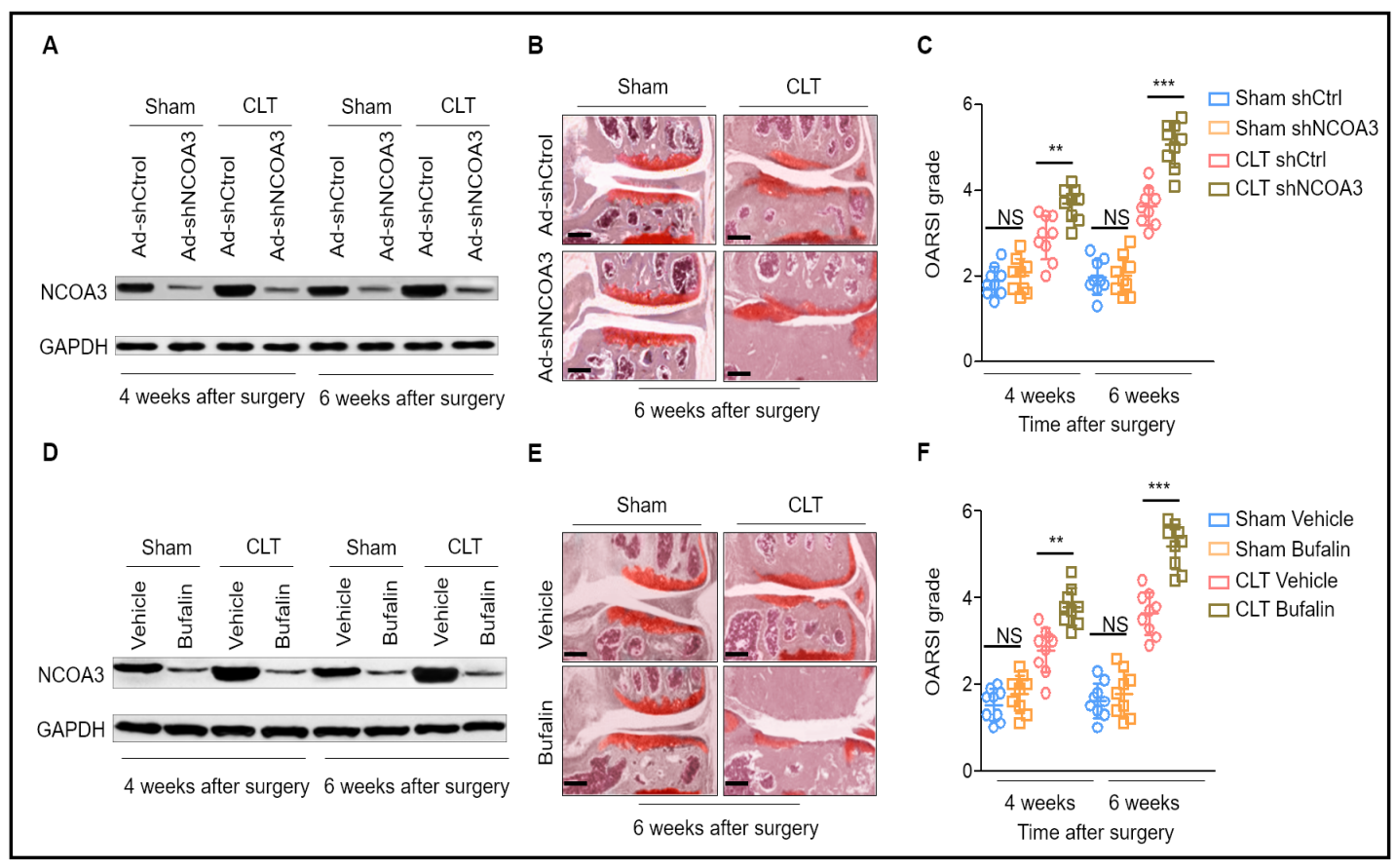

Fig. 5. NCOA3 inhibition promotes posttraumatic OA progression in vivo (A-C) 8-week-old male C57BL/6 wild type mouse knees were intra-articularly injected with Ad-shCtrl or Ad-shNCOA3 3 days after CLT or sham surgery and then sacrificed at 4 or 6 weeks after surgery. Each group contained 9 mice. (A) Western blot analysis of protein level of NCOA3 in mouse articular chondrocytes. (B) Knees were sectioned and stained with Hematoxylin-Safranin O. (C) Cartilage damage was scored according to OARSI histological grading scale. (D-E) 8-week-old male C57BL/6 wild type mouse knees were intra-articularly injected with DMSO or bufalin $(1 \mathrm{mg} / \mathrm{kg})$ three times per week after CLT or sham surgery and then sacrificed at 4 or 6 weeks after surgery. Each group contained 9 mice. (D) Western blot analysis of protein level of NCOA3 in mouse articular chondrocytes. (E) Knees were sectioned and stained with Hematoxylin-Safranin O. (F) Cartilage damage was scored according to OARSI histological grading scale. In (A) and (D), 4 mice included in each group were randomly selected and the representative results were shown. GAPDH was used as loading control. In (B) and (E), Scale bar, $200 \mu \mathrm{m}$. Shown here were the representative results of 9 mice included in each group at 6 weeks after surgery. In (C) and (F), each symbol represented the value of OARSI grade for each mouse. The results in shNCOA3 group were compare to those of Ad-shCtrl group at each time point as indicated. The statistical analysis was performed using Student's t test. Data are mean \pm s.d. ${ }^{* * *}$, $\mathrm{P}<0.001 ; * *, \mathrm{P}<0.01 ; \mathrm{NS}$, not significant.

5B) and the OARSI grade was higher (Fig. 5C), in contrast to shCtrl group. These results point out that NCOA3 loss is able to accelerate posttraumatic OA progression. In addition, we also applied bufalin to inhibit NCOA3 expression in cartilage in vivo (Fig. 5D), similar to the above findings, compared with vehicle control, bufalin treatment group again displayed more severe cartilage damage (Fig. 5E) and higher OARSI grade (Fig. 5F) at 4 and 6 weeks post-surgery, further strengthening our conclusion that NCOA3 lose promotes posttraumatic OA progression.

\section{NCOA3 loss exacerbates NF- $\kappa B$ pathway activation in posttraumatic $O A$}

Several possible molecular mechanisms were proposed for explaining the roles of NCOA3 in OA, such as regulating skeletal metabolism or cartilage homeostasis in a hormone-dependent fashion [7, 8]. Apparently, much of these speculations are based on the consideration that NCOA3, a nuclear receptor coactivator, could directly bind nuclear receptors and is involved in their coactivation when responding to different hormones [32], however, none of which have been clearly validated or reported. The data presented in Fig. 
S3 suggest that NCOA3 deficiency may not be a prerequisite for OA initiation, which alludes to an indirect manner by which NCOA3 may connect to OA. Thus, to understand how NCOA3 deficiency impinges on posttraumatic OA progression, other than investigating its direct elicited effects, we turned to focus on the indirect effect NCOA3 may exert on transcriptional activity downstream of signaling pathways which are known critical for OA development. In our posttraumatic OA model, we found that the NF- $\mathrm{BB}$ pathway was activated in CLT group compared with sham group, as evidenced by the upregulation of its known downstream

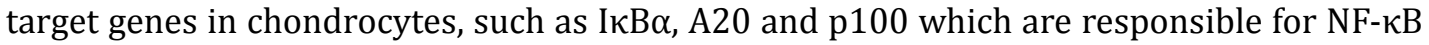
feedback regulation, and molecules functioning in inflammation, extracellular matrix (ECM), stress and adhesion involved in OA pathology (Fig. 6A). Surprisingly, in the meantime, NCOA3 deficiency in chondrocytes resulted in the enhancement of transcriptional induction of these genes (Fig. 6A), indicating that NF- $\kappa$ B pathway activation was exacerbated when NCOA3 was deficient. This result was further confirmed by the elevated levels of phosphorylation of

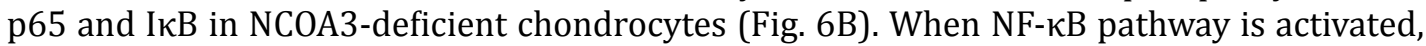
transcriptional factor NF- $\kappa B$ will translocate from cytoplasm into nucleus for fulfilling its

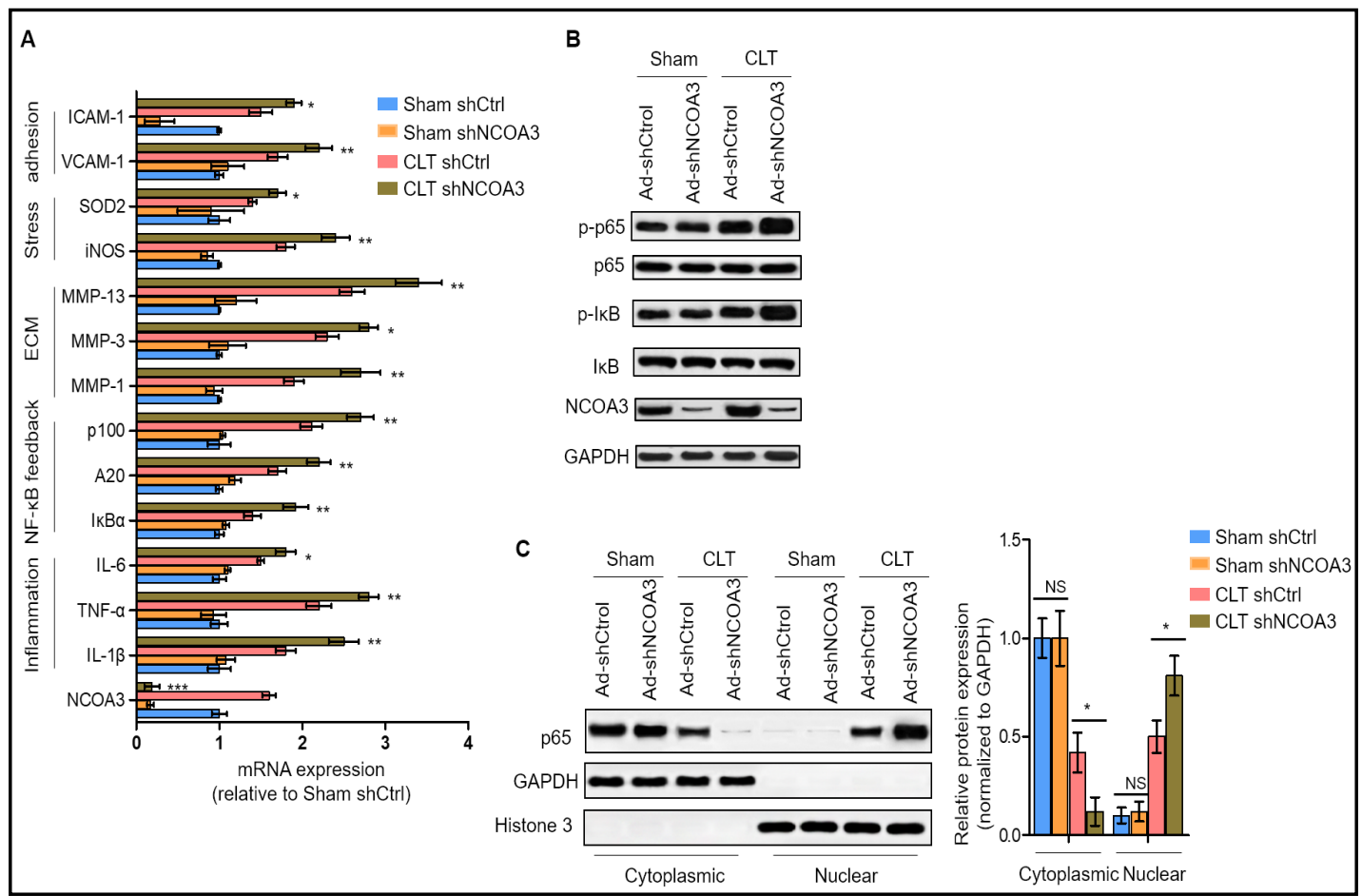

Fig. 6. NCOA3 inhibition exacerbates NF- $\mathrm{NB}$ signaling pathway activation in posttraumatic OA(A-C) Mice were treated as in Fig. 5. (A-C). (A) Real-time quantitative PCR analysis of mRNA level of genes as indicated. Expression level of each gene was normalized to human GAPDH. The results were expressed as relative to those of Sham shCtrl group. Each column represents the mean value of 3 replicates for each sample and the results were representative of 4 chondrocyte donors from each group at 6 weeks after surgery. Data in CTL shNCOA3 were compared to CLT shCtrl. (B) Western blot analysis of protein level as indicated in mouse articular chondrocytes. 4 mice included in each group were randomly selected and the representative results were shown. GAPDH was used as loading control. (C) Western blot analysis of protein level of p65 in cytoplasmic and nuclear faction of chondrocytes. GAPDH and Histone 3 were used as cytoplasmic and nuclear loading control, respectively. 4 mice included in each group were randomly selected and the representative results were shown (Left). The statistical analysis of band intensity of Western blot results was shown (Right). The protein expression in each sample was normalized to human GAPDH and the result relative to Sham shCtrl group was shown. Data in shNCOA3 group were compared to those of shCtrl group as indicated. The statistical analysis was performed using Student's $t$ test. Data are mean \pm s.d. ${ }^{* * *}, \mathrm{P}<0.001$; **, $\mathrm{P}<0.01 ; *, \mathrm{P}<.0 .05$; NS, not significant.

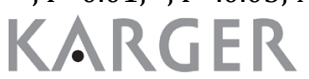


function [33]. Indeed, in contrast to sham group, NF- $\mathrm{BB}$ shuttled into nucleus when NF$\kappa \mathrm{B}$ pathway was activated in CLT group, and as expected, even more proportion of NF- $\kappa \mathrm{B}$ was present in nucleus faction in NCOA3-deficient chondrocytes (Fig. 6C). These results demonstrate that NCOA3 loss exacerbates the functional activation of NF- $\mathrm{KB}$ pathway in posttraumatic $\mathrm{OA}$.

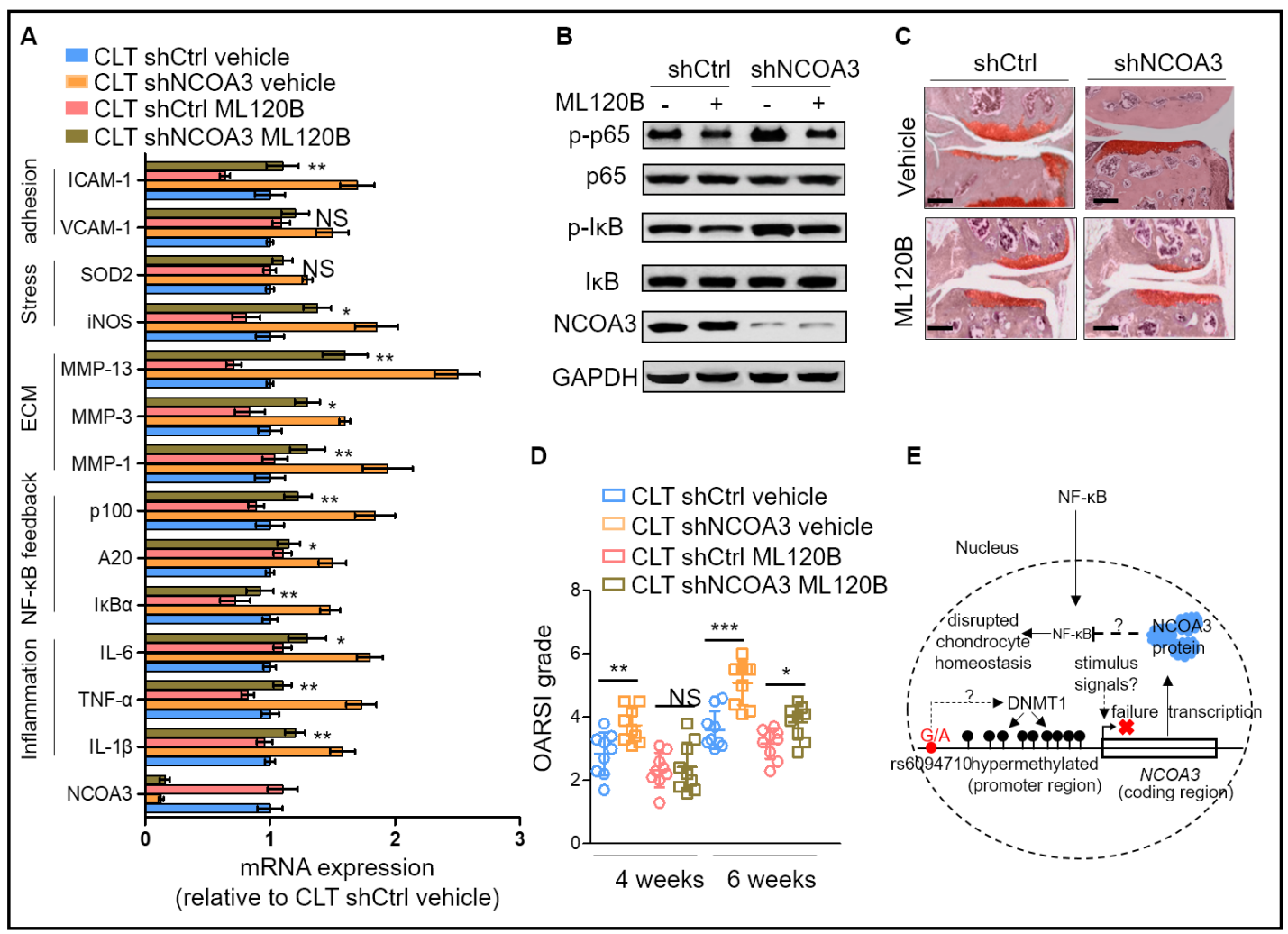

Fig. 7. NCOA3 inhibition promotes posttraumatic OA partially by enhancing NF- $\mathrm{KB}$ signaling pathway activation(A-D) 8-week-old male C57BL/6 wild type mouse knees were intra-articularly injected with AdshCtrl or Ad-shNCOA3 3 days after CLT surgery and also administrated orally with $60 \mathrm{mg} / \mathrm{kg}$ ML120B in $0.5 \%$ hydroxypropyl-methylcellulose $/ 0.2 \%$ Tween or this vehicle twice daily and then sacrificed at 4 or 6 weeks after surgery. Each group contained 9 mice. (A) Real-time quantitative PCR analysis of mRNA level of genes as indicated. Expression level of each gene was normalized to human GAPDH. The results were expressed as relative to those of CLT shCtrl vehicle group. Each column represents the mean value of 3 replicates for each sample and the results were representative of 4 chondrocyte donors from each group at 6 weeks after surgery. Data in CTL shNCOA3 ML120B were compared to those of CLT shNCOA3 vehicle group. (B) Western blot analysis of protein level as indicated in mouse articular chondrocytes. 4 mice included in each group at 6 weeks after surgery were randomly selected and the representative results were shown. GAPDH was used as loading control. (C) Knees were sectioned and stained with Hematoxylin-Safranin O. Scale bar, $200 \mu \mathrm{m}$. Shown here were the representative results of 9 mice included in each group at 6 weeks after surgery. (D) Cartilage damage was scored according to OARSI histological grading scale. Each symbol represented the value of OARSI grade for each mouse. The results in shNCOA3 group were compare to those of shCtrl group at each time point as indicated. (E) The proposed mode of mechanisms that underlies the role of NCOA3 in delaying OA development. The statistical analysis was performed using Student's t test. Data are mean \pm s.d. ${ }^{* * *}, \mathrm{P}<0.001 ; * *, \mathrm{P}<0.01 ;{ }^{*}, \mathrm{P}<.0 .05$; NS, not significant. 


\section{Cellular Physiology Cell Physiol Biochem 2018;49:2396-2413

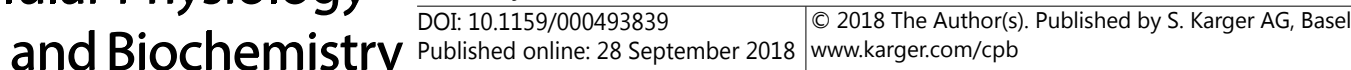 \\ Kang et al.: Effects of NCOA3 Loss on Chondrocytes and Osteoarthritis}

NCOA3 loss promotes posttraumatic osteoarthritis partially by exacerbating NF- $\kappa B$ pathway activation

Next, to establish a causative link between NCOA3 inhibition-dependent NF- $\kappa \mathrm{B}$ activation and exacerbated posttraumatic OA progression, we used ML120B, one potent and selective inhibitor of IKK $\beta$ kinase $[34,35]$, to inhibit NF- $\mathrm{KB}$ pathway activation. In posttraumatic $\mathrm{OA}$ model created by CLT surgery, ML120B treatment alone reduced the transcription of certain target genes downstream of NF- $\kappa B$ signaling pathway (Fig. 7A) and phosphorylation of p65

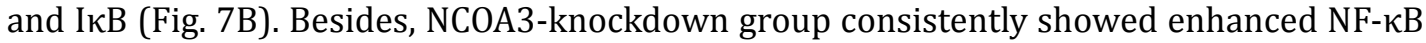
signaling activation, and these enhanced effects of NCOA3-knockdown on NF- $\kappa B$ signaling activation were largely dampened by ML120B treatment (Fig. 7A-7B), overall demonstrating its efficient inhibitory function on NF- $\kappa \mathrm{B}$ signaling in vivo. Along with the alleviated NF- $\kappa \mathrm{B}$ signaling activation caused by ML120B treatment, the difference of cartilage damage severity (Fig. 7C) and OARSI grade (Fig. 7D) between shCtrl and shNCOA3 group were significantly reduced, although not totally recovered to the level of CLT shCtrl group. These evidence support a critical role of NCOA3 inhibition-dependent NF- $\mathrm{KB}$ activity in posttraumatic OA progression, and indicate that the stimulating effect of NCOA3 deficiency on posttraumatic $\mathrm{OA}$ progression is at least partially dependent on the enhanced NF- $\mathrm{BB}$ signaling activation.

Taken together, these data shown in present study propose a working model wherein chondrocytes with rs6094710 SNP have higher DNMT1-mediated hypermethylation spreading over promoter region of NCOA3 gene, resulting in its transcriptional induction failure, and conversely, its normal upregulation responding to stimulus signals would otherwise serve to restrain NF- $\mathrm{KB}$ signaling activation for maintaining chondrocyte signature and homeostasis (Fig. 7E). Thus, we provide a causal explanation for the association between rs6094710 SNP and higher risk of developing OA. The raised key questions such as how rs6094710 SNP affects DNMT1-mediated activity on NCOA3 gene, which stimulus signals are responsible for inducing NCOA3 transcription, and how NCOA3 interferes NF- $\kappa B$ signaling in the context of OA pathology remain to be elucidated (Fig. 7E).

\section{Discussion}

In this study, we reveal the defective ability of upregulating NCOA3 in chondrocytes carrying rs6094710 SNP in both mRNA and protein levels by examining clinical knee OA cartilage and in vitro dedifferentiated chondrocytes. We further show that this NCOA3 induction failure is associated with abnormal DNMT1-mediated hypermethylation which is caused by rs6094710 SNP upstream of NCOA3 gene promoter region. In functional studies, NCOA3 performs an important role in maintaining the molecular signature of chondrocytes in vitro, however, NCOA3 seems dispensable for preventing OA initiation, since NCOA3 loss alone cannot trigger $\mathrm{OA}$ in young mice. However, the loss of NCOA3 promotes the progression of posttraumatic OA. Mechanistically, NF- $\mathrm{B}$ pathway is excessively activated in the absent of NCOA3, and the promotive effect of NCOA loss on posttraumatic OA progression is significantly alleviated when administrated with NF- $\kappa B$ pathway inhibitor, indicating that NCOA3 loss promotes posttraumatic OA at least partially by enhancing NF- $\kappa B$ pathway activation (Fig. 7E).

We observed less NCOA3 expression in OA chondrocytes carrying rs6094710 SNP compared with normal OA counterparts. These results appear consistent with previous study [18], further supporting the expression pattern of $N C O A 3$ gene in this setting. Nevertheless, we did not find reduced NCOA3 expression in OA cartilage (Fig. 1D-1F), as reported by Evangelou et al. [7]. We suppose that this incompatibility may derive from the difference of grouping design where we stratified OA patients by genotype at rs6094710 SNP. Actually, the evidence obtained from these two individual cohorts instead highlight the impairment of NCOA3 upregulation when rs6094710 SNP exists, which was not noticed in precious studies, and is very likely to account for the above distinct expression pattern of $N C O A 3$ gene we have seen, since normal cells are able to upregulate NCOA3, whereas 


\section{Cellular Physiology Cell Physiol Biochem 2018;49:2396-2413

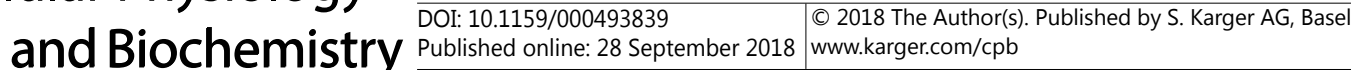 \\ Kang et al.: Effects of NCOA3 Loss on Chondrocytes and Osteoarthritis}

NCOA3 in cells with rs6094710 SNP remain nearly steady. Although we further provide in vitro evidence to strengthen this notion, more examinations and related studies with larger clinical samples are needed to testify it. On the other hand, except for knee OA we analyzed, further investigations are needed to test whether the expression pattern of NCOA3 can be extended to other type of OA.

DNA methyltransferase-meidated DNA methylation is a reversible epigenetic modification that modulates gene expression without influencing the gene sequence [36]. One recent research [10] has suggested an association between the dysregulation of NCOA3 expression caused by rs6094710 SNP with DNA methylation modulation. Our study confirms this association and further uncovers DNTM1 as an important executor to perform this role. Because the maintenance of genomic methylation patterns is primarily mediated by DNMT1 [37], therefore it may not be so surprising if DNA methylation mechanism is required for $N C O A 3$ gene regulation. The intriguing issue is that how a single G/A transition SNP influences DNMT1 activity. We suppose this SNP probably functions through enhancing the interaction between DNMT1 and target genomic sites, as evidenced by our findings that the binding intensity of DNMT1 and NCOA3 promoter maintains nearly unchanged in cells with rs6094710 SNP during dedifferentiation, which would otherwise gradually fade in normal cells (Fig. S1C). DNMT1 is consists of a replication foci-targeting domain (RFD), a DNAbinding CXXC domain, a pair of bromo-adjacent homology (BAH) domains and a C-terminal catalytic domain [37]. Presumably, after binding to this mutated locus, the affinity of CXXC domain or maybe other partners of DNMT1 is altered, and DNMT1 becomes more accessible to NCOA3 gene. The elucidation of the specific mechanism underlying this modulation warrants further experiments.

According to our evidence, the maintenance of certain level of NCOA3 appears essential for chondrocytes to attenuate OA-like dedifferentiation and catabolic events triggered by IL-1 $\beta$ in vitro. However, since these conditions do not faithfully equate with OA model, the effect of NCOA3 on chondrocyte differentiation and its contribution to OA needs further investigations by utilizing other favorable experimental models, such as NCAO3 knockout mice. As a transcriptional coactivator, NCOA3 has been shown to directly regulate the transcription of matrix metalloproteinase (MMP)-2 and MMP-13 through its coactivation of AP-1 and PEA3 [38]. Therefore, direct transcriptional regulation is a possible mechanism. Another potential mechanism is through indirectly mediating transcriptional effect emitting from upstream signal. However, the signals and the downstream pathways that regulate the transcriptional activity of NCOA3 in chondrocytes remain largely unknown. In primary B cells, NCOA3 can be activated by the signal transducer and activator of transcription 6 (STAT6) and enhances its transcriptional activity through formation of a complex composed of STAT6, p300/CBP and NCOA3 [39]. Its appealing to test whether this signal axis or complex is responsible for the regulatory role of NCOA3 in maintaining chondrocyte identity. Furthermore, what makes condition more puzzling is that this mechanism seems inapplicable for in vivo scenario, since chondrocytes do not lose their molecular characteristics or develop OA when NCOA3 is deficient in young mice. One speculation is the interference imposed by the functional redundancy among the family member of NCOAs (i.e., NCOA3 and NCOA1) [32]. Furthermore, whether NCOA3 loss affects the development of age-related OA needs further investigations in proper animal models.

Transcriptional factor NF- $\kappa \mathrm{B}$ can be activated by pro-inflammatory cytokines, stressrelated factors and ECM degradation products, and induce the expression of genes which cause articular joint destruction and finally lead to OA initiation and progression [33, 40]. Hence, targeting NF- $\kappa B$ signaling has now presented as novel potential therapeutic strategy for OA treatment [41]. Considering the results that NCOA3 loss promotes the progression of posttraumatic OA and this effect is associate with amplified NF- $\mathrm{BB}$ pathway, its plausible that NCOA3 may play a retarding role in OA progression by constraining inflammatory response mediated by NF- $\kappa B$ pathway in chondrocytes where a local inflammatory milieu has been already established, for example, knee inflammation occurring in posttraumatic OA model we used in this study. More direct evidence, especially those from clinical human 


\section{Cellular Physiology Cell Physiol Biochem 2018;49:2396-2413 \begin{tabular}{l|l|l|l|l} 
DOI: 10.1159/000493839 & $\begin{array}{l}\text { C) } 2018 \text { The Author(s). Published by S. Karger AG, Basel } \\
\text { www.karger.com/cpb }\end{array}$
\end{tabular} \\ Kang et al.: Effects of NCOA3 Loss on Chondrocytes and Osteoarthritis}

samples, are needed for examining the correlation between NCOA3 and inflammation surveillance. Since we only examined the effect of NCOA3 inhibition on posttraumatic OA, further observations of the ectopic overexpression of NCOA3 in cartilage or transgenic mice expressing $\mathrm{NCOA}_{3}$ may be helpful to directly determine whether NCOA3 protects against posttraumatic OA progression. Besides, to our knowledge at present, how NCOA3 directly affects NF- $\kappa B$ signaling still remain largely unknown. In-depth studies clarifying the mechanisms underlying how NCOA3 regulates NF- $\kappa \mathrm{B}$ pathway and exerts an anti-catabolic role in posttraumatic OA pathogenesis would help us to deeply understand the role of NCOA3 in $\mathrm{OA}$ pathogenesis.

\section{Acknowledgements}

This research was supported by the fund of Natural Science Foundation of Hebei Province (H2012206068).

\section{Disclosure Statement}

No conflict of interests exists.

\section{References}

1 Messier SP, Beavers DP, Herman C, Hunter DJ, DeVita P: Are unilateral and bilateral knee osteoarthritis patients unique subsets of knee osteoarthritis? A biomechanical perspective. Osteoarthr Cartilage 2016;24:807-813.

-2 Cutolo M, Berenbaum F, Hochberg M, Punzi L, Reginster JY: Commentary on recent therapeutic guidelines for osteoarthritis. Semin Arthritis Rheu 2015;44:611-617.

-3 Pap T, Korb-Pap A: Cartilage damage in osteoarthritis and rheumatoid arthritis--two unequal siblings. Nat Rev Rheumatol 2015;11:606-615.

-4 Bijlsma JW, Berenbaum F, Lafeber FP: Osteoarthritis: an update with relevance for clinical practice. Lancet 2011;377:2115-2126.

-5 Castano-Betancourt MC, Evans DS, Ramos YF, Boer CG, Metrustry S, Liu Y, den Hollander W, van Rooij J, Kraus VB, Yau MS, Mitchell BD, Muir K, Hofman A, Doherty M, Doherty S, Zhang W, Kraaij R, Rivadeneira F, Barrett-Connor E, Maciewicz RA, et al.: Thickness and Hip Osteoarthritis. PLoS Genet 2016;12:e1006260.

-6 Zeggini E, Panoutsopoulou K, Southam L, Rayner NW, Day-Williams AG, Lopes MC, Boraska V, Esko T, Evangelou E, Hoffman A, Houwing-Duistermaat JJ, Ingvarsson T, Jonsdottir I, Jonnson H, Kerkhof HJ, Kloppenburg M, Bos SD, Mangino M, Metrustry S, Slagboom PE, et al: Identification of new susceptibility loci for osteoarthritis (arcOGEN): a genome-wide association study. Lancet 2012;380:815-823.

7 Evangelou E, Kerkhof HJ, Styrkarsdottir U, Ntzani EE, Bos SD, Esko T, Evans DS, Metrustry S, Panoutsopoulou K, Ramos YF, Thorleifsson G, Tsilidis KK, arc OC, Arden N, Aslam N, Bellamy N, Birrell F, Blanco FJ, Carr A, Chapman K, et al.: A meta-analysis of genome-wide association studies identifies novel variants associated with osteoarthritis of the hip. Ann Rheum Dis 2014;73:2130-2136.

-8 Tsezou A: Osteoarthritis year in review 2014: genetics and genomics. Osteoarthr Cartilage 2014;22:20172024.

9 Rushton MD, Gee FH, Reynard LN, Loughlin J: The osteoarthritis association marked by SNP rs6094710 mediates its effect by reducing the expression of NCOA3 in joint tissues. Osteoarthr Cartilage 2014;22:S237.

10 Villalvilla A, Aubourg G, Deehan D, Loughlin J, Reynard LN: DNA methylation modulated by the osteoarthritis susceptibility locus marked by rs6094710 is associated with regulation of NCOA3 gene expression. Osteoarthr Cartilage 2017;25:S207-S208. 


\section{Cellular Physiology Cell Physiol Biochem 2018;49:2396-2413

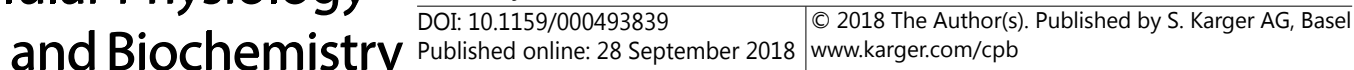

11 Altman R, Asch E, Bloch D, Bole G, Borenstein D, Brandt K, Christy W, Cooke TD, Greenwald R, Hochberg M, Howell D, Kaplan D, Koopman W, Longley S, Mankin H, McShane J, Medsger T, Meenan R, Mikkelsen W, Moskowitz R, et al.: Development of criteria for the classification and reporting of osteoarthritis. Classification of osteoarthritis of the knee. Diagnostic and Therapeutic Criteria Committee of the American Rheumatism Association. Arthritis Rheum 1986;29:1039-1049.

12 Kellgren JH, Lawrence JS: Radiological assessment of osteo-arthrosis. Ann Rheum Dis 1957;16:494-502.

13 Monteagudo S, Cornelis FMF, Aznar-Lopez C, Yibmantasiri P, Guns LA, Carmeliet P, Cailotto F, Lories RJ: DOT1L safeguards cartilage homeostasis and protects against osteoarthritis. Nat Commun 2017;8:15889.

14 Zhou G, Hashimoto Y, Kwak I, Tsai SY, Tsai MJ: Role of the Steroid Receptor Coactivator SRC-3 in Cell Growth. Mol Cell Biol 2003;23:7742-7755.

-15 Ferrell WR, Lockhart JC, Kelso EB, Dunning L, Plevin R, Meek SE, Smith AJ, Hunter GD, McLean JS, McGarry F, Ramage R, Jiang L, Kanke T, Kawagoe J: Essential role for proteinase-activated receptor-2 in arthritis. J Clin Invest 2003;111:35-41.

16 Glasson SS, Chambers MG, Van Den Berg WB, Little CB: The OARSI histopathology initiative recommendations for histological assessments of osteoarthritis in the mouse. Osteoarthr Cartilage 2010;18:S17-23.

17 Ruan MZ, Dawson B, Jiang MM, Gannon F, Heggeness M, Lee BH: Quantitative imaging of murine osteoarthritic cartilage by phase-contrast micro-computed tomography. Arthritis Rheum 2013;65:388-396.

18 Gee F, Rushton MD, Loughlin J, Reynard LN: Correlation of the osteoarthritis susceptibility variants that map to chromosome 20q13 with an expression quantitative trait locus operating on NCOA3 and with functional variation at the polymorphism rs116855380. Arthritis Rheum 2015;67:2923-2932.

19 Aigner T, Soder S, Gebhard PM, McAlinden A, Haag J: Mechanisms of disease: role of chondrocytes in the pathogenesis of osteoarthritis--structure, chaos and senescence. Nat Clin Pract Rheum 2007;3:391-399.

-20 Ma B, Leijten JC, Wu L, Kip M, van Blitterswijk CA, Post JN, Karperien M: Gene expression profiling of dedifferentiated human articular chondrocytes in monolayer culture. Osteoarthr Cartilage 2013;21:599603.

-21 Benya PD, Padilla SR, Nimni ME: Independent regulation of collagen types by chondrocytes during the loss of differentiated function in culture. Cell 1978;15:1313-1321.

22 Huang J, Wang Y, Guo Y, Sun S: Down-regulated microRNA-152 induces aberrant DNA methylation in hepatitis B virus-related hepatocellular carcinoma by targeting DNA methyltransferase 1. Hepatology 2010;52:60-70.

-23 Ning X, Shi Z, Liu X, Zhang A, Han L, Jiang K, Kang C, Zhang Q: DNMT1 and EZH2 mediated methylation silences the microRNA-200b/a/429 gene and promotes tumor progression. Cancer Lett 2015;359:198205.

24 Percharde M, Azuara V: Essential roles for the nuclear receptor coactivator Ncoa3 in pluripotency. Cell Cycle 2013;12:195-196.

25 Percharde M, Lavial F, Ng JH, Kumar V, Tomaz RA, Martin N, Yeo JC, Gil J, Prabhakar S, Ng HH, Parker MG, Azuara V: Ncoa3 functions as an essential Esrrb coactivator to sustain embryonic stem cell self-renewal and reprogramming. Genes Dev 2012;26:2286-2298.

26 Wu Z, Yang M, Liu H, Guo H, Wang Y, Cheng H, Chen L: Role of nuclear receptor coactivator 3 (Ncoa3) in pluripotency maintenance. J Biol Chem 2012;287:38295-38304.

-27 Yan J, Tsai SY, Tsai MJ: SRC-3/AIB1: transcriptional coactivator in oncogenesis. Acta pharmacol Sin 2006;27:387-394.

28 Wang Y, Lonard DM, Yu Y, Chow DC, Palzkill TG, Wang J, Qi R, Matzuk AJ, Song X, Madoux F, Hodder P, Chase P, Griffin PR, Zhou S, Liao L, Xu J, O'Malley BW: Bufalin is a potent small-molecule inhibitor of the steroid receptor coactivators SRC-3 and SRC-1. Cancer Res 2014;74:1506-1517.

29 Zou Z, Luo X, Nie P, Wu B, Zhang T, Wei Y, Wang W, Geng G, Jiang J, Mi Y: Inhibition of SRC-3 enhances sensitivity of human cancer cells to histone deacetylase inhibitors. Biochem Bioph Res Co 2016;478:227233.

-30 Kapoor M, Martel-Pelletier J, Lajeunesse D, Pelletier JP, Fahmi H: Role of proinflammatory cytokines in the pathophysiology of osteoarthritis. Nat Rev Rheum 2011;7:33-42.

-31 Ruan MZ, Erez A, Guse K, Dawson B, Bertin T, Chen Y, Jiang MM, Yustein J, Gannon F, Lee BH: Proteoglycan 4 expression protects against the development of osteoarthritis. Sci Transl Med 2013;5:176ra134. 


\section{Cellular Physiology Cell Physiol Biochem 2018;49:2396-2413

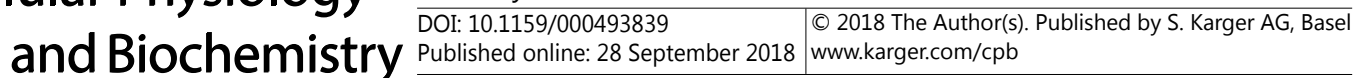

Kang et al.: Effects of NCOA3 Loss on Chondrocytes and Osteoarthritis

32 Xu J, Liao L, Ning G, Yoshida-Komiya H, Deng C, O’Malley BW: The steroid receptor coactivator SRC-3 (p/ CIP/RAC3/AIB1/ACTR/TRAM-1) is required for normal growth, puberty, female reproductive function, and mammary gland development. P Natl Acad Sci USA 2000;97:6379-6384.

33 Rigoglou S, Papavassiliou AG: The NF-kappaB signalling pathway in osteoarthritis. Int J Biochem Cell B 2013;45:2580-2584.

-34 Nagashima K, Sasseville VG, Wen D, Bielecki A, Yang H, Simpson C, Grant E, Hepperle M, Harriman G, Jaffee B, Ocain T, Xu Y, Fraser CC: Rapid TNFR1-dependent lymphocyte depletion in vivo with a selective chemical inhibitor of IKKbeta. Blood 2006;107:4266-4273.

-35 Izmailova ES, Paz N, Alencar H, Chun M, Schopf L, Hepperle M, Lane JH, Harriman G, Xu Y, Ocain T, Weissleder R, Mahmood U, Healy AM, Jaffee B: Use of molecular imaging to quantify response to IKK-2 inhibitor treatment in murine arthritis. Arthritis Rheum 2007;56:117-128.

-36 Feng J, Zhou Y, Campbell SL, Le T, Li E, Sweatt JD, Silva AJ, Fan G: Dnmt1 and Dnmt3a maintain DNA methylation and regulate synaptic function in adult forebrain neurons. Nat Neurosci 2010;13:423-430.

-37 Song J, Rechkoblit 0, Bestor TH, Patel DJ: Structure of DNMT1-DNA complex reveals a role for autoinhibition in maintenance DNA methylation. Science 2011;331:1036-1040.

-38 Yan J, Erdem H, Li R, Cai Y, Ayala G, Ittmann M, Yu-Lee LY, Tsai SY, Tsai MJ: Steroid receptor coactivator-3/ AIB1 promotes cell migration and invasiveness through focal adhesion turnover and matrix metalloproteinase expression. Cancer Res 2008;68:5460-5468.

39 Arimura A, vn Peer M, Schroder AJ, Rothman PB: The transcriptional co-activator p/CIP (NCoA-3) is upregulated by STAT6 and serves as a positive regulator of transcriptional activation by STAT6. J Biol Chem 2004;279:31105-31112.

40 Olivotto E, Otero M, Marcu KB, Goldring MB: Pathophysiology of osteoarthritis: canonical NF-kappaB/ IKKbeta-dependent and kinase-independent effects of IKKalpha in cartilage degradation and chondrocyte differentiation. RMD Open 2015;1:e000061.

41 Saito T, Tanaka S: Molecular mechanisms underlying osteoarthritis development: Notch and NF-kappaB. Arthritis Res Ther 2017;19:94. 